\title{
Effect of Smoking Cessation and Reduction on the Risk of Cancer in Korean Men: A Population Based Study
}

\section{Seulggie Choi, MD \\ Jooyoung Chang, $\mathrm{BSC}^{2}$ \\ Kyuwoong Kim, BSC \\ Sang Min Park, MD, PhD, MPH \\ Kiheon Lee, MD3,4}

${ }^{1}$ Department of Biomedical Sciences, Seoul National University College of Medicine, Seoul, ${ }^{2}$ Seoul National University College of Medicine, Seoul, ${ }^{3}$ Department of Family Medicine, Seoul National University Hospital, Seoul, ${ }^{4}$ Department of Family Medicine, Seoul National University Bundang Hospital, Seongnam, Korea

Correspondence: Kiheon Lee, MD

Department of Family Medicine, Seoul National University Bundang Hospital, Seoul National

University College of Medicine, 82 Gumi-ro 173 beon-gil, Bundang-gu, Seongnam 13620, Korea Tel: 82-31-787-7801

Fax: 82-31-787-4834

E-mail: keyhoney@gmail.com

Received July 9, 2017

Accepted November 20, 2017

Published Online November 24, 2017

*Seulggie Choi and Jooyoung Chang contributed equally to this work.

\begin{abstract}
Purpose
The purpose of this study was to investigate the effect of smoking habit change on the risk
\end{abstract} of cancer.

\section{Materials and Methods}

From the Korean National Health Insurance Service database, we determined the change in smoking habit between the first (2002 and 2003) and second (2004 and 2005) health examination periods. A total of 143,071 men were categorized into baseline heavy $(\geq 20$ cigarettes per day), moderate (10-19 cigarettes per day), light ( $<10$ cigarettes per day) smokers, quitters, and never smokers, after which the change in smoking status was determined during the second health examination. The participants were then followed up from 2006 to 2013 for all cancer, smoking related cancer, and lung cancer.

\section{Results}

Compared to heavy continual smokers, heavy smokers who quit had reduced risk of smoking related cancer (hazard ratio [HR], 0.74; 95\% confidence interval [Cl], 0.56 to 0.97) and tended to have reduced risk of all cancer ( $\mathrm{HR}, 0.87 ; 95 \% \mathrm{Cl}, 0.75$ to 1.00). Moderate smokers who reduced the amount of smoking to light levels had decreased risk of all cancer (HR, $0.82 ; 95 \% \mathrm{Cl}, 0.72$ to 0.94 ), smoking related cancer (HR, 0.74; $95 \% \mathrm{Cl}, 0.59$ to 0.93 ), and lung cancer (HR, $0.55 ; 95 \% \mathrm{Cl}, 0.38$ to 0.79 ) compared to heavy continual smokers.

\section{Conclusion}

Smoking reduction decreases the risk of all cancer, smoking related cancer, and lung cancer. While smoking cessation should be the treatment of choice for smokers, smoking reduction may serve as an alternative strategy for those who cannot quit.

Key words

Smoking cessation, Smoking reduction, Neoplasms

\section{Introduction}

Smoking is associated with a wide range of serious illnesses including cardiovascular disease [1], stroke [2], and cancer [3]. Among them, cancer has age-standardized rates for incidence and mortality of 290.5 and 87.9 per 100,000 in Korea in 2013, respectively [4], highlighting the importance of determining the role of modifiable risk factors for cancer. Therefore, the changes in smoking habits, which include smoking cessation and reduction, play a crucial role in altering the risk of cancer and determining the risk reducing effect of smoking habit change on cancer merits further research.

Smoking cessation has been proven to reduce the risk of various types of cancer [5-7]. However, smoking cessation methods have had limited success, with many smokers unable to abstain from tobacco use for longer than one year [8]. Due to the low success rate of smoking cessation, smoking reduction, decreasing the amount of cigarettes smoked 
per day, has been proposed as an alternate strategy to reduce the risk of cancer [9]. Previous studies demonstrating the risk reducing effect of smoking reduction on cancer only investigated lung cancer and was based on a Western population [10] or a specific population of Korean civil servants aged between 30 and 58 years [11]. Particularly, civil servants tend to be younger and have better overall health than the general population that is most susceptible to the development of cancer. Therefore, studies determining the effect of smoking reduction on the risk of cancer in a general Asian population are needed.

Recently, the Korean National Health Insurance Service (NHIS) developed a cohort of Korean citizens who underwent general health screenings from 2002 to 2013, called the National Health Insurance Service-National Health Screening Cohort (NHIS-HealS) [12,13]. As all Korean citizens aged 40 years or more enrolled in the NHIS undergo biannual general health examinations, NHIS-HealS has the advantage of representing the general population well. Therefore, we conducted a longitudinal study to determine the association between smoking habit change and the risk of developing cancer using the NHIS-HealS database.

\section{Materials and Methods}

\section{Study population}

The Korean NHIS provides mandatory health insurance for Korean citizens covering nearly all forms of health care services [14], including biannual health examinations for all dependents aged 40 years or older [12]. Overall, $65.3 \%$ of those eligible participate in the health examinations [15]. The NHIS-HealS database includes data from hospital admissions, outpatient department visits, the Korean diagnosisrelated group (DRG) code, and pharmaceutical prescriptions. Data on demographic profiles, death registries, disability registries, and health examinations were merged with the claims data and anonymized with strict confidentiality guidelines. Numerous previous reports have used the NHIS-HealS database to conduct epidemiologic studies, and its validity has been demonstrated elsewhere $[13,16,17]$.

Among 179,390 male participants aged between 40 and 82 years who underwent health examinations in both the first (2002 and 2003) and second (2004 and 2005) periods, 6,140 smoking initiators (those who were never smokers during the first period and current smokers during the second period) and 4,295 relapsers (those who were quitters during the first period and current smokers during the second period) were excluded. Then, 12,073 men who were diag- nosed with cancer before the index date of 1 January 2006 were excluded. Participants who died $(n=500)$ and with missing values on covariates $(n=468)$ were further excluded. Finally, in order to reduce the possibility of reverse-causality, in which having worsening health conditions may have prompted smoking cessation or reduction, 12,843 participants diagnosed with cancer within the first 4 years of follow-up (from 1 January 2006 to 31 December 2009) were excluded. Ultimately, 143,071 men were followed up from 1 January 2006 to 31 December 2013 for newly diagnosed cancer. A list of items included in reports of cohort studies according to the STROBE checklist can be found in the supplementary materials (S1 Table).

\section{Cancer events}

The primary outcome was newly diagnosed all cancer, smoking related cancer, and lung cancer, which was determined by the 10th Revision of the International Classification of Diseases (ICD-10) code. All cancer was defined as any ICD-10 code starting with C (malignant neoplasms). Smoking related cancer was defined according to the 2014 Surgeon's General Report, which included malignancies of the head and neck (ICD-10 code C00-C14), esophagus (C15), stomach (C16), colorectum (C18-C20), hepatocellular carcinoma (C22.0), pancreas (C25), larynx (C32), trachea (C33), bronchus and lung (C34), bladder (C67), kidney, kidney pelvis, or ureter (C64-C66, C68), or acute myeloid leukemia (C92.0) [18].

We defined an event of newly diagnosed cancer when a participant visited the hospital with a diagnosis of an ICD10 code for cancer and met any of the following additional criteria: (1) made at least three outpatient visits with the same ICD-10 code, (2) was hospitalized for two or more days with the code, (3) received any cancer treatments based on the DRG code for malignancy, or (4) died due to causes related to the code. The date of cancer diagnosis was the first hospital visit date among newly diagnosed cancer patients.

\section{Smoking habit change and covariates}

Participants were grouped according to the baseline smoking status determined by a questionnaire during the first health examination into heavy smokers ( $\geq 20$ cigarettes per day), moderate smokers (10-19 cigarettes per day), light smokers ( $<10$ cigarettes per day), quitters, and never smokers. Using the questionnaire from the second health examination, smokers were categorized into continual smokers and quitters. Sustained quitters and never smokers were participants who were quitters and never smokers for both health examination periods, respectively. Ultimately, the study population was categorized into heavy continual 


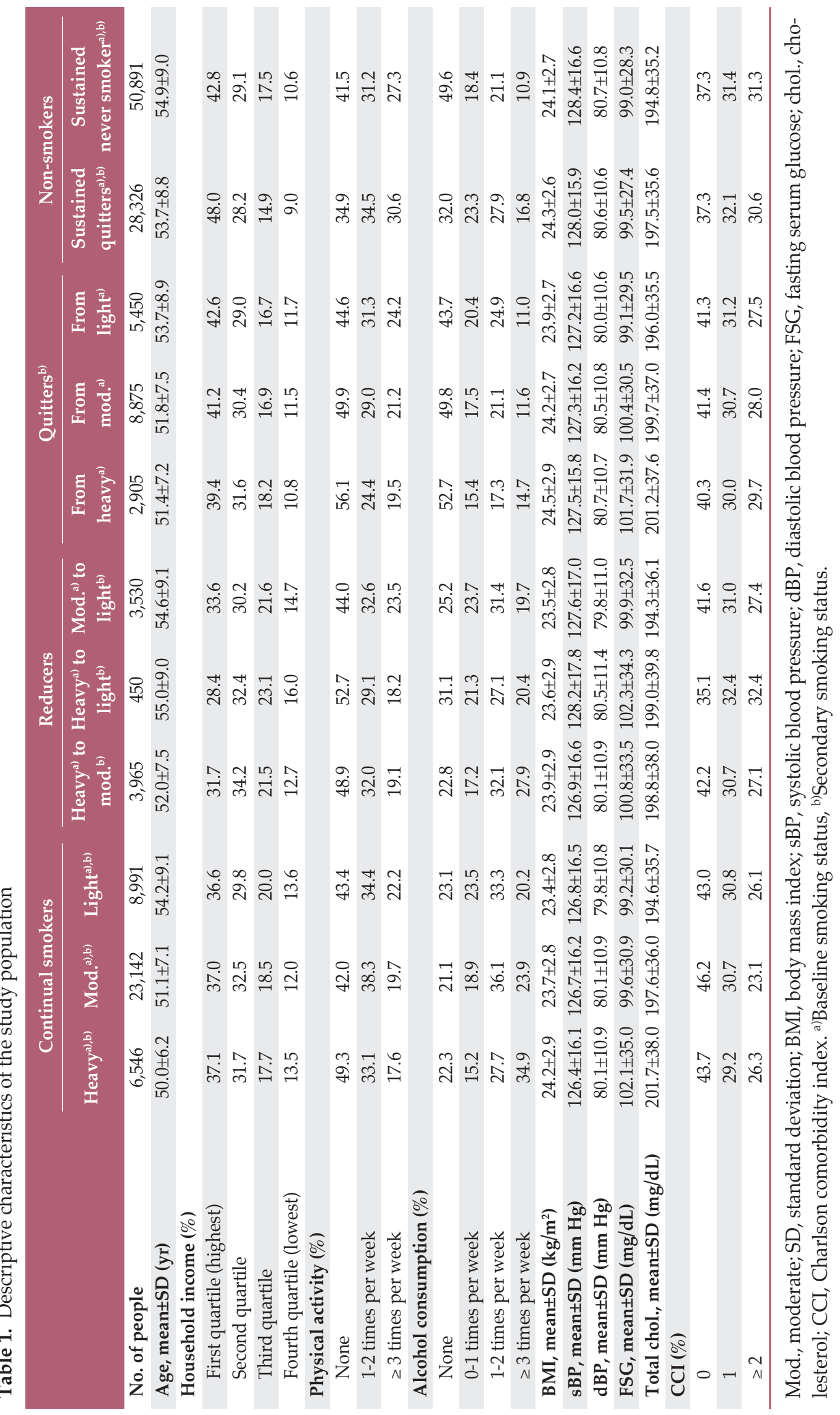


smokers, moderate continual smokers, light continual smokers, heavy reducers to moderate levels, heavy reducers to light levels, moderate reducers to light levels, heavy quitters, moderate quitters, light quitters, sustained quitters, and sustained never smokers.

Covariates, extracted between 2002 and 2005, included age, household income, physical activity, alcohol consumption, total cholesterol levels, blood pressure, fasting serum glucose, body mass index (BMI), and Charlson comorbidity index (CCI). Household income was categorized according to each patient's insurance premium status. CCI was calculated using the ICD-10 code diagnoses for major comorbidities between 2002 and 2005 [19].

\section{Statistical analysis}

We performed Cox proportional hazards regression analyses to estimate hazard ratios (HRs) and 95\% confidence intervals (CIs) between smoking habit change groups relative to heavy continual smokers for cancer. The assumption of proportionality for the Cox model was graphically tested and verified using the Schoenfeld residual method. Smoking reduction was defined as the reduction in smoking amount between heavy ( $\geq 20$ cigarettes per day), moderate (10-19 cigarettes per day), and light ( $<10$ cigarettes per day) amounts. HRs were adjusted for age (continuous, years), household income (categorical, first, second, third, and fourth quartiles), physical activity (categorical, $0,1-2$, and 3 or more times per week), alcohol consumption (categorical, 0, 0-1, 1-2, and 3 or more times per week), BMI (continuous, $\mathrm{kg} / \mathrm{m}^{2}$ ), systolic blood pressure (continuous, $\mathrm{mm} \mathrm{Hg}$ ), diastolic blood pressure (continuous, $\mathrm{mm} \mathrm{Hg}$ ), fasting serum glucose (continuous, $\mathrm{mg} / \mathrm{dL}$ ), total cholesterol (continuous, $\mathrm{mg} / \mathrm{dL}$ ), and CCI (continuous).

Statistical significance was defined as a p-value of less than 0.05 in a two-sided manner. We performed all statistical analyses using STATA ver. 13.0 (STATA Corp., College Station, TX).

\section{Ethical statement}

The study protocol was approved by the Institutional Review Board (IRB) of Seoul National University Bundang Hospital (IRB number: X-1701/378-902). The requirement for participant consent was waived as the NHIS-HealS database is anonymized in adherence to strict confidentiality guidelines.

\section{Results}

A total of 13,612 newly diagnosed cancer events were observed during 1,102,254 person-years. The distribution of the study participants for each baseline characteristic respective to smoking habit change is shown in Table 1. Compared to heavy continual smokers, sustained never smokers tended to be older, have higher household income, exercise more, consume less alcohol, and have higher CCI values. Compared to heavy continual smokers, heavy smokers who quit tended to have higher household income, exercise less, consume alcohol less, and have higher CCI values.

Table 2 shows the effect of smoking habit change on the risk of developing all cancer, smoking related cancer, and lung cancer. Compared to heavy continual smokers, moderate continual smokers and light continual smokers had decreased risk of all cancer (HR, 0.85 ; 95\% CI, 0.78 to 0.94 for moderate and HR, 0.88 ; $95 \%$ CI, 0.79 to 0.97 for light), smoking related cancer (HR, $0.79 ; 95 \% \mathrm{CI}, 0.67$ to 0.93 for moderate and $\mathrm{HR}, 0.69 ; 95 \% \mathrm{CI}, 0.57$ to 0.84 for light), and lung cancer (HR, $0.63 ; 95 \%$ CI, 0.49 to 0.81 for moderate and HR, 0.49; 95\% CI, 0.37 to 0.66 for light) in a dose-responsive manner. Similarly, sustained quitters and sustained never smokers had decreased risk of all cancer (HR, $0.79 ; 95 \% \mathrm{CI}, 0.73$ to 0.87 for quitters and $\mathrm{HR}, 0.76$; $95 \% \mathrm{CI}, 0.70$ to 0.83 for never smokers), smoking related cancer (HR, $0.60 ; 95 \% \mathrm{CI}, 0.51$ to 0.71 for quitters and HR, 0.47 ; $95 \% \mathrm{CI}, 0.40$ to 0.55 for never smokers), and lung cancer (HR, $0.34 ; 95 \% \mathrm{CI}, 0.26$ to 0.44 for quitters and HR, $0.25 ; 95 \% \mathrm{CI}, 0.19$ to 0.32 for never smokers) in a dose-responsive manner.

Compared to heavy continual smokers, heavy quitters had decreased risk of smoking related cancer (HR, $0.74 ; 95 \% \mathrm{CI}$, 0.56 to 0.97 ) and tended to have reduced risk of all cancer (HR, 0.87, 95\% CI, 0.75 to 1.00). Furthermore, moderate smokers who reduced the amount of cigarettes to light levels had reduced risk of all cancer (HR, $0.82 ; 95 \% \mathrm{CI}, 0.72$ to 0.94 ), smoking related cancer (HR, $0.74 ; 95 \% \mathrm{CI}, 0.59$ to 0.93$)$, and lung cancer (HR, $0.55 ; 95 \%$ CI, 0.38 to 0.79 ).

\section{Discussion}

In this large-scale longitudinal study of Korean men, we have shown that smoking reduction decreased the risk of developing all cancer, smoking related cancer, and lung cancer. The results of this study add to previous findings $[10,11]$ demonstrating the risk reducing effect of smoking reduction on lung cancer by showing that smoking reduction decreased the risk of not only lung cancer but also all cancer 


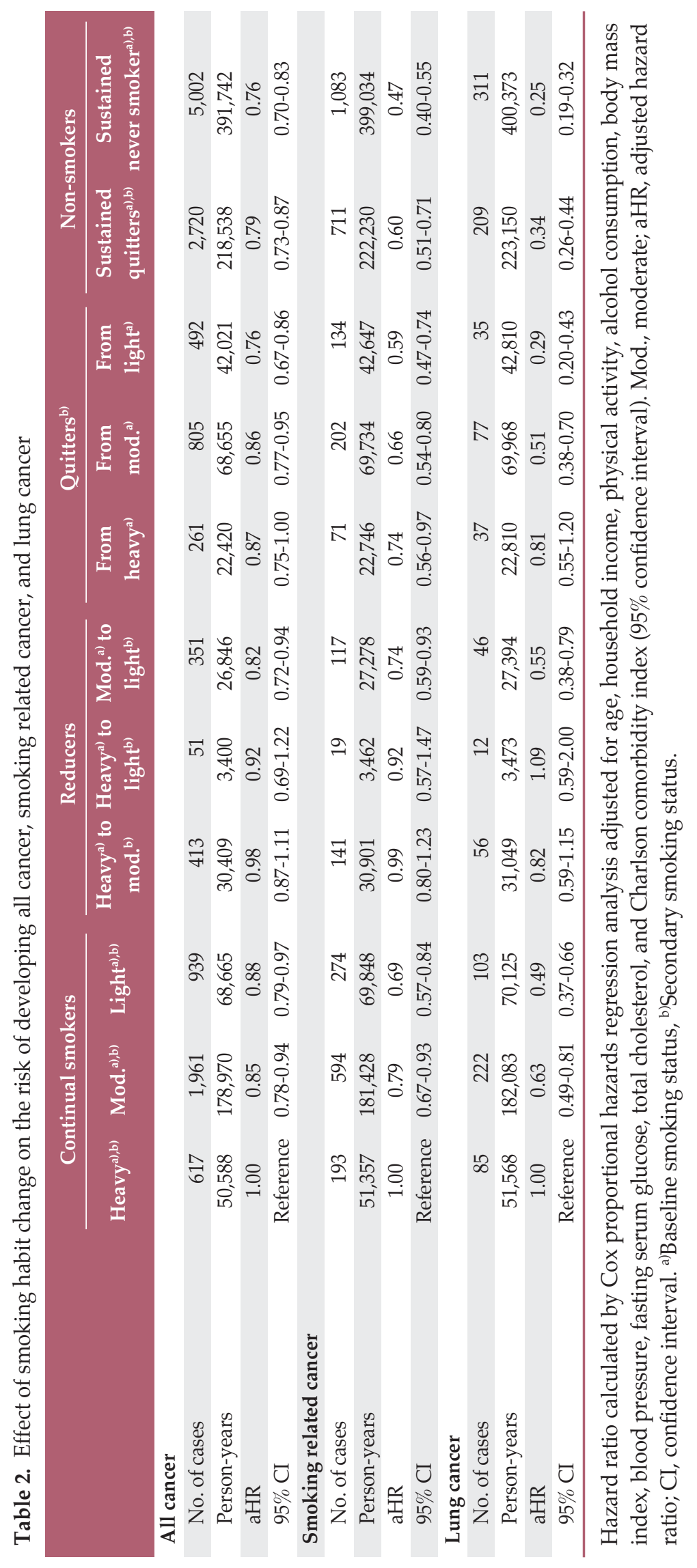


and smoking related cancer among a large Asian population that represents the general population well.

Recently, two cohort studies have investigated the association between smoking reduction and cancer. In 2005, Godtfredsen and colleagues have shown that compared to continual heavy smokers, reduction of the smoking amount by $50 \%$ led to decreased risk of lung cancer (HR, 0.73; 95\% CI, 0.54 to 0.98 ) among 19,714 Danish men and women [10]. Similarly, in 2008, Song and colleagues have shown that reducing the amount of smoking from heavy to moderate (HR, 0.72; 95\% CI, 0.49 to 0.89 ) and heavy to light (HR, 0.63; $95 \%$ CI, 0.46 to 0.84 ) led to decreased risk of lung cancer among 479,156 Korean civil servant men aged between 30 and 58 years [11]. Our results also demonstrate that smoking reduction led to decreased risk of lung cancer. Furthermore, we have also shown that smoking reduction was beneficial for decreasing the risk of all cancer and smoking related cancer.

Contrary to the results from the study by Song and colleagues [11], smoking reduction was associated with decreased risk of not only lung cancer but also all cancer and smoking related cancer in our study. There are several possible explanations for this discrepancy. First, while the study populations from both studies are comprised of Korean men, our study population is composed those aged between 40 and 82 years (compared to between 30 and 58 years) regardless of employment status (compared to civil servants). As age is an important factor in determining the risk of cancer and employment is directly associated with general health status, the innate differences in the study populations in terms of age and employment may have contributed to the significant benefit of smoking reduction for all cancer and smoking related cancer in our results.

While moderate reducers to light levels had decreased risk of cancer, there was a lack of significant risk-reduction among heavy smokers who reduced the amount of cigarettes to moderate and light amounts, which is another difference in our results compared to previous findings $[10,11]$. There are several possible explanations for this finding. First, reducers may have failed to maintain the reduced amount of cigarettes after the second health examination and relapsed to the amount originally smoked [20]. A previous study examining carcinogenic biomarkers in reducers showed that only two out of 23 smokers were able to maintain their smoking reduction habits beyond 12 weeks [21]. Second, even with successful reduction, the intensity at which the reducer inhales his or her cigarette may compensate for the decrease in the number of cigarettes [22]. This concept, known as compensatory smoking, is based on previous studies that revealed smoking reduction was not associated with a corresponding reduction in tobacco-related carcinogenic biomarkers [21]. Therefore, factors such as failure to maintain the reduced amount and compensatory smoking may have contributed to the lack of significant risk-reducing benefit of smoking reduction when comparing individual groups.

Several limitations must be considered when interpreting the results from our study. First, the responses from the smoking questionnaire may not accurately reflect the participants' actual smoking status, leading to incorrect categorization of smoking status groups. Second, the follow-up duration of eight years may not be sufficient to completely reveal the effects of smoking habit change. Particularly, as cancer is a slowly-developing chronic disorder, a longer follow-up duration may be needed to fully determine the effect of smoking reduction and cessation on the risk of cancer. Therefore, future studies with longer follow-up durations are needed to validate the findings of this study.

Finally, the event of newly diagnosed cancer was based on the health claims data, which may be less accurate compared to cancer events defined by the National Cancer Registry. Nevertheless, a recent study comparing the incidence rates of cancer between the National Cancer Registry and NHIS data in Korea has shown that the incidence rate for cancer using the NHIS data (363.1 per 100,000 people) was similar to that from the National Cancer Registry (361.9 per 100,000 people) [23]. Furthermore, another previous study comparing the correlation of breast cancer events between the claims data and national cancer registry data in Japan showed positive predictive values of breast cancer events defined by the claims data ranging from $65.8 \%$ to $90.7 \%$ [24]. Therefore, while future studies using the National Cancer Registry data are needed, previous studies showing high correlation between cancer events from the NHIS and National Cancer Registry data suggest that using the claims data for defining cancer events is appropriate.

Smoking reduction decreases the risk of all cancer, smoking related cancer, and lung cancer. While smoking cessation should be considered the primary management strategy for smokers, smoking reduction may be considered an alternate method of management for those who cannot quit in order to reduce the risk of developing cancer.

\section{Electronic Supplementary Material}

Supplementary materials are available at Cancer Research and Treatment website (http:// www.e-crt.org).

\section{Conflicts of Interest}

Conflict of interest relevant to this article was not reported. 


\section{Acknowledgments}

We would like to thank Mi Hee Cho, Hye-Yeon Koo, and Ji-Hye Jun for their input on the study design and interpretation of the results, as well as Hyeyoung Yoo for administrative support. This study used NHIS-HealS data (NHIS-2017-2-472) from the Korean NHIS. This study was not funded.

\section{References}

1. Ambrose JA, Barua RS. The pathophysiology of cigarette smoking and cardiovascular disease: an update. J Am Coll Cardiol. 2004;43:1731-7.

2. Wolf PA, D'Agostino RB, Kannel WB, Bonita R, Belanger AJ. Cigarette smoking as a risk factor for stroke. The Framingham Study. JAMA. 1988;259:1025-9.

3. Sasco AJ, Secretan MB, Straif K. Tobacco smoking and cancer: a brief review of recent epidemiological evidence. Lung Cancer. 2004;45 Suppl 2:S3-9.

4. Jung KW, Won YJ, Oh CM, Kong HJ, Lee DH, Lee KH, et al. Cancer statistics in Korea: incidence, mortality, survival, and prevalence in 2014. Cancer Res Treat. 2017;49:292-305.

5. Ebbert JO, Yang P, Vachon CM, Vierkant RA, Cerhan JR, Folsom AR, et al. Lung cancer risk reduction after smoking cessation: observations from a prospective cohort of women. J Clin Oncol. 2003;21:921-6.

6. Altieri A, Bosetti C, Talamini R, Gallus S, Franceschi S, Levi F, et al. Cessation of smoking and drinking and the risk of laryngeal cancer. Br J Cancer. 2002;87:1227-9.

7. Mulder I, Hoogenveen RT, van Genugten ML, Lankisch PG, Lowenfels $\mathrm{AB}$, de Hollander $\mathrm{AE}$, et al. Smoking cessation would substantially reduce the future incidence of pancreatic cancer in the European Union. Eur J Gastroenterol Hepatol. 2002;14:1343-53.

8. Hughes JR, Carpenter MJ. Does smoking reduction increase future cessation and decrease disease risk? A qualitative review. Nicotine Tob Res. 2006;8:739-49.

9. Shiffman S, Gitchell JG, Warner KE, Slade J, Henningfield JE, Pinney JM. Tobacco harm reduction: conceptual structure and nomenclature for analysis and research. Nicotine Tob Res. 2002;4 Suppl 2:S113-29.

10. Godtfredsen NS, Prescott E, Osler M. Effect of smoking reduction on lung cancer risk. JAMA. 2005;294:1505-10.

11. Song YM, Sung J, Cho HJ. Reduction and cessation of cigarette smoking and risk of cancer: a cohort study of Korean men. J Clin Oncol. 2008;26:5101-6.

12. Seong SC, Kim YY, Khang YH, Park JH, Kang HJ, Lee H, et al. Data resource profile: The National Health Information Database of the National Health Insurance Service in South Korea. Int J Epidemiol. 2017;46:799-800.

13. Lee J, Lee JS, Park SH, Shin SA, Kim K. Cohort profile: The
National Health Insurance Service-National Sample Cohort (NHIS-NSC), South Korea. Int J Epidemiol. 2017;46:e15.

14. Kwon S. Payment system reform for health care providers in Korea. Health Policy Plan. 2003;18:84-92.

15. Korean National Health Insurance Corporation. Analysis of 2009 national health screening program. Wonju: Korean National Health Insurance Corporation; 2010.

16. Park SM, Yun YH, Kim YA, Jo M, Won YJ, Back JH, et al. Prediagnosis body mass index and risk of secondary primary cancer in male cancer survivors: a large cohort study. J Clin Oncol. 2016;34:4116-24.

17. Seong SC, Kim YY, Park SK, Khang YH, Kim HC, Park JH, et al. Cohort profile: the National Health Insurance ServiceNational Health Screening Cohort (NHIS-HEALS) in Korea. BMJ Open. 2017;7:e016640.

18. Warren GW, Alberg AJ, Kraft AS, Cummings KM. The 2014 Surgeon General's report: "The health consequences of smoking: 50 years of progress": a paradigm shift in cancer care. Cancer. 2014;120:1914-6.

19. Sundararajan V, Henderson T, Perry C, Muggivan A, Quan H, Ghali WA. New ICD-10 version of the Charlson comorbidity index predicted in-hospital mortality. J Clin Epidemiol. 2004;57:1288-94.

20. Godtfredsen NS, Holst C, Prescott E, Vestbo J, Osler M. Smoking reduction, smoking cessation, and mortality: a 16-year follow-up of 19,732 men and women from The Copenhagen Centre for Prospective Population Studies. Am J Epidemiol. 2002;156:994-1001.

21. Hurt RD, Croghan GA, Wolter TD, Croghan IT, Offord KP, Williams GM, et al. Does smoking reduction result in reduction of biomarkers associated with harm? A pilot study using a nicotine inhaler. Nicotine Tob Res. 2000;2:327-36.

22. Hatsukami DK, Kotlyar M, Allen S, Jensen J, Li S, Le C, et al. Effects of cigarette reduction on cardiovascular risk factors and subjective measures. Chest. 2005;128:2528-37.

23. Seo HJ, Oh IH, Yoon SJ. A comparison of the cancer incidence rates between the national cancer registry and insurance claims data in Korea. Asian Pac J Cancer Prev. 2012;13:6163-8.

24. Sato I, Yagata H, Ohashi Y. The accuracy of Japanese claims data in identifying breast cancer cases. Biol Pharm Bull. 2015;38:53-7. 